1. Walsh, P.C., and Donker, P.J. 1982. Impotence following radical prostatectomy: insight into etiology and prevention. J. Urol. 128:492-497.

2. Kattan, M.W., Eastham, J.A., Stapleton, A.M., Wheeler, T.M., and Scardino, P.T. 1998. A preoperative nomogram for disease recurrence following radical prostatectomy for prostate cancer. J. Natl. Cancer Inst. 90:766-771.

3. D’Amico, A.V., et al. 1999. Pretreatment nomogram for prostate-specific antigen recurrence after radical prostatectomy or external-beam radiation therapy for clinically localized prostate cancer. J. Clin. Oncol. 17:168-172.

4. Glinsky, G.V., Glinskii, A.B., Stephenson, A.J., Hoffman, R.M., and Gerald, W.L. 2004. Gene expression profiling predicts clinical outcome of prostate cancer. J. Clin. Invest. 113:913-923. doi:10.1172/ JCI200420032.

5. Glinsky, G.V., Ivanova, Y.A., and Glinskii, A.B. 2003.
Malignancy-associated regions of transcriptional activation: gene expression profiling identifies common chromosomal regions of recurrent transcriptional activation in human prostate, breast, ovarian and colon cancers. Neoplasia. 5:221-228.

6. Kattan, M.W., Wheeler, T.M., and Scardino, P.T. 1999. Postoperative nomogram for disease recurrence after radical prostatectomy for prostate cancer. J. Clin. Oncol. 17:1499-1507.

\title{
The immune response to AIDS virus infection: good, bad, or both?
}

\author{
Steven G. Deeks ${ }^{1}$ and Bruce D. Walker ${ }^{2}$
}

\begin{abstract}
${ }^{1}$ Department of Medicine, University of California, San Francisco, San Francisco, California, USA. ${ }^{2}$ Howard Hughes Medical Institute and Partners AIDS Research Center, Massachusetts General Hospital and Division of AIDS, Harvard Medical School, Boston, Massachusetts, USA.
\end{abstract}

\begin{abstract}
A potent antigen-specific $T$ cell response to HIV infection can contribute to the control of viral replication and is therefore beneficial to the host. However, HIV-mediated increases in generalized $\mathrm{T}$ cell activation also appear to accelerate both viral replication and $\mathrm{CD} 4^{+} \mathrm{T}$ cell depletion. A new study in the JCI attempts to experimentally distinguish the beneficial versus harmful aspects of this immune response (see the related article beginning on page 836).
\end{abstract}

Acute HIV infection is associated with symptoms almost everyone has experienced - fever, sore throat, swollen glands, and often a transient rash. These are not specific to HIV, but rather a consequence of the high level of immune activation that is induced in defense of HIV and other acute viral infections. Although a strong immune response is presumably beneficial in most acute infections, specific properties of HIV raise the question as to whether immune activation in this setting may also have harmful consequences. HIV selectively infects and replicates in activated CD4 cells, suggesting that the antiviral effects of the acute immune response may be counterbalanced by the detrimental effects of adding additional fuel (i.e., activated CD4 $\mathrm{T}$ cells) to the fire. The proinflammatory aspects of HIV infection also result in the activation and proliferation of $\mathrm{CD}^{+} \mathrm{T}$ cells specific for antigens other than HIV (the so-called "innocent bystanders"), which can also become productively infected with HIV, albeit at a lower frequency (1). Thus, a stronger immune response to HIV might have the paradoxical effect of

Nonstandard abbreviation used: simian immunodeficiency virus (SIV).

Conflict of interest: The authors have declared that no conflict of interest exists.

Citation for this article:

J. Clin. Invest. 113:808-810 (2004).

doi:10.1172/JCI200421318. enhancing viral replication and accelerating disease progression.

Unfortunately, this synergistic interaction between immune activation and viral replication is only part of the story. More importantly from the host's perspective, chronic heightened activation of the immune system may also contribute in a direct manner to progressive $\mathrm{CD}^{+} \mathrm{T}$ cell depletion. One widely accepted model of HIV immunopathogenesis postulates that heightened immune and proliferation of memory-effector $\mathrm{CD}^{+}$ $T$ cells (2). These cells are destined to die rapidly as a consequence of activationinduced cell death and/or due to direct infection by HIV. Over time, the naive and central memory pools become exhausted and unable to generate new primary responses or maintain the peripheral $\mathrm{CD}^{+}$ $\mathrm{T}$ cell count $(3,4)$.

Given this background, some have questioned whether the immune response to HIV might cause more harm than good. Indeed, substantial circumstantial evidence for such paradoxical effects exists, including the observation that immune activation is a strong independent predictor of disease outcome in antiretroviraluntreated and -treated individuals $(5,6)$. Along these lines, much has recently been made of the observation that sooty mangabeys, the natural host of simian immunodeficiency virus (SIV), support activation results in accelerated activation high levels of viral replication but fail to exhibit a clear increase in immune activation and/or inflammation $(7,8)$. These animals appear to tolerate SIV infection quite well, having life spans that are not dramatically different from those of uninfected animals. In contrast, when SIV is experimentally transferred to rhesus macaques, dramatic increases in immune activation occur, and animals progress rapidly to AIDS and death.

\section{Separating the protective versus destructive aspects of the immune response}

In this issue of the JCI, Garber and colleagues use a clever experimental approach in an attempt to differentiate the potential harmful aspects of virusmediated increases in $\mathrm{T}$ cell activation and/or proliferation from the benefits associated with the generation of an effective SIV-specific T cell response (9). Four adult rhesus macaques were treated with a monoclonal antibody combination designed to block costimulation and thereby eliminate cellular activation during acute SIV infection. CTLA4Ig was used to block CD4 stimulation through CD28CD80/86 interactions, and anti-CD40L was administered to block CD4 stimulation through CD40-CD40L interactions. The effect of this treatment, administered before and during acute SIV infection, was compared to the effect of no treatment in four acutely infected control animals. Viral load, $T$ cell activation, antigen-specific $T$ cell and $B$ cell immune responses, and the emergence of CTL escape mutations were closely monitored during the first few months of infection. 
A

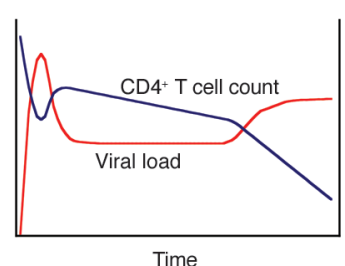

Time

Figure 1

Schematic figure illustrating the impact of fitness and pathogenicity on virus-host outcomes. (A) Highly fit, pathogenic virus: HIV-1 in most antiretroviral-untreated humans and SIV in rhesus macaques is both highly fit (i.e., replicates at high levels) and highly pathogenic (i.e., causes $T$ cell depletion and disease). (B) Poorly fit virus: highly pathogenic viruses may cause limited disease if replication is limited. HIV-1 under successful antiretroviral treatment and HIV-1 being controlled immunologically are examples of environments whereby HIV-1 is unable to replicate efficiently and is therefore poorly fit. Most therapeutic strategies are aimed at reducing the capacity of HIV-1 to replicate efficiently. (C) Less pathogenic virus: SIV in sooty mangabeys replicates efficiently and is therefore highly fit. This virus, however, does not consistently cause disease, suggesting that its pathogenic effect has been attenuated. There has been only a limited amount of investigation aimed at reducing the pathogenic effect of HIV-1 in humans.

So what was the effect of blocking cellular activation? Costimulation blockade, albeit incomplete, resulted in lower levels of proliferating $\mathrm{CD}^{+} \mathrm{T}$ cells, and lower levels of peak viremia (9). However, it also reduced the magnitude of virus-specific CD8 T cell responses (as defined by the number of SIVspecific IFN- $\gamma$ T cells) and dramatically altered the antigen-specific $B$ cell response (as defined by the generation of neutralizing activity against SIVmac251, a related lab-adapted strain of SIV). Collectively, these decreases in the magnitude and presumably breadth of the adaptive immune response significantly impaired the subsequent ability of the immune system to control viral replication. Interestingly, those same antibody-treated animals experienced less early immune escape as measured by polymorphisms within known CD8 T cell epitopes, consistent with reduced immune selection pressure.

Despite transient costimulation blockade, AIDS virus infection was not transformed into a benign condition in these animals (9). After costimulation blockade was discontinued, SIV replication increased in a transient manner. Viral and T cell dynamics during the immediate post-blockade period were consistent with an early increase in proliferating cells (a surrogate for infectable cells) followed by a possible increase in the SIV-specific T cell and B cell immune responses. SIV RNA levels increased and then decreased as an apparent consequence. Interestingly, despite all of the early manipulations, the steadystate level of viremia that occurred at or after week 14 of the study was similar in the experimental versus control animals.

\section{Viral fitness and pathogenicity}

What novel pathogenesis insights were realized as a consequence of this study? On one hand, the immunogenicity of SIV (or, by extension, HIV) increases the number of proliferating $\mathrm{CD}^{+} \mathrm{T}$ cells, which contributes in a direct manner to early peak viremia (9). On the other hand, the data indicate that generation of an effective SIVspecific immune response is a critical factor in curtailing viral replication. Thus, where control of viral replication is the desired outcome, the immune response is indeed both beneficial and harmful. These studies provide the first clear evidence in primates of the contribution of cellular activation to disease enhancement.

This study raises the question of whether disease outcome can be modified by decreasing the proinflammatory aspects of the infection. As mentioned above, viral replication in sooty mangabeys is often high, yet these animals do not progress clinically (10). While viral fitness - a measure of the virus's capacity to replicate in vivo - is similar in macaques and sooty mangabeys, viral pathogenicity - a measure of the capacity of a virus to cause disease - clearly differs between the two species (Figure 1). This difference appears to be mediated by reduced levels of immune activation and $\mathrm{T}$ cell turnover in the sooty mangabey $(7,11)$. Thus, preservation of $\mathrm{CD}^{+} \mathrm{T}$ cells rather than establishment of a lower viral load set-point may be the more relevant outcome for studies in which immune activation is targeted. The work of Garber et al. focused on virologic rather than immunologic outcomes, per- haps because the sample size required to assess the latter outcome would be much larger (9). The fact that the treated animals appeared to progress clinically more rapidly than the untreated animals suggests, however, that transient costimulation blockade did not provide any durable protection, but rather on balance accelerated disease progression.

\section{Why costimulation blockade was not protective}

Several factors may have contributed to the failure of the treated animals to achieve a durable clinical and immunologic benefit. First, the administration of costimulation blockade may have been too short. An interesting follow-up experiment would be to continue costimulation blockade indefinitely to determine whether immunologic and clinical health could have been maintained in a situation where virus replicates at will but generalized immune activation is prevented.

Second, costimulation blockade may have blunted the generation of beneficial SIV-specific immune responses without affecting the harmful SIV-mediated increases in generalized immune activation. Garber and colleagues provide some evidence for this, as their treated animals clearly had an early increase in T cell proliferation (as measured by Ki67 expression) despite costimulation blockade (9). Since the mechanism for the SIV/HIV-mediated increase in immune activation remains unclear, interventions that effectively prevent an antigen-specific immune response (e.g., costimulation blockade) may not necessarily prevent the deleterious impact $\mathrm{SIV} / \mathrm{HIV}$ has on immune activation and by extension $\mathrm{CD}^{+} \mathrm{T}$ cell loss and clinical progression. For example, as suggested by Garber and colleagues, SIV/HIV-mediated depletion of regulatory or suppressor $\mathrm{T}$ cells may be the central factor. Rapid depletion of these cells in vivo by the cytopathic effect of HIV/SIV could result in loss of immunologic control and high $\mathrm{T}$ cell activation and/or turnover. Understanding the mechanism whereby SIV/HIV causes nonspecific increases in immune activation is clearly needed if specific interventions to block this effect are to occur.

Third, the SIV-infected macaque may not be an appropriate model in which to test the hypothesis that the immune response might be more harmful that beneficial. Discussion on this point requires speculation on how viral evolution within a host 
impacts on viral evolution within a population. Theoretically, SIV/HIV and their infected hosts may co-evolve towards a point where virus replication and transmission efficiency increase while the capacity of the virus to cause disease decreases. A state of maximal viral fitness with minimal pathogenicity may already have been achieved in sooty mangabeys, the natural host of SIV. To what degree this has or will occur in humans remains speculative. However, recent data clearly indicate that HIV has evolved within genetically distinct populations to avoid immune detection (12-14). Preliminary data suggest that this immune evasion has been associated with higher viral load set-points (i.e., greater fitness) and an accelerated rate of disease progression (i.e., greater or at least preserved pathogenicity) $(12,14)$. Evolutionary pressure leading to a benign sooty mangabey-like virus/host relationship in humans may require selective pressure at the level of the host rather than or in addition to the virus (15).

The preceding discussion raises a potential methodological concern with regard to the use of macaques by Garber and colleagues. Since macaques as a species have not been previously exposed to SIV, the virus has had little chance to evolve under immune selection pressure, and a very potent and effective SIV-specific immune response would be expected. Therapies aimed at suppressing this immune response would likely have relatively severe deleterious effects. A similar experiment performed in a species where the virus has already partially adapted (e.g., humans) may have a very different outcome. Determining the proper role - if any - of agents aimed at suppressing non-specific immune activation as an adjunct to antiretroviral therapy should remain a focus of clinical investigation, and the model presented by Garber et al. may be particularly useful.

\section{Role of immune modulators in the clinical management of HIV}

Do the data presented in the paper by Garber et al. (9) have potential clinical implications? There remains continued interest in manipulating the immune system to control HIV replication in the absence of antiretroviral therapy, mostly focused on augmentation of the adaptive immune responses via therapeutic vaccines or brief antiretroviral treatment interruptions. However, the use of cyclosporin as an adjunct to highly active antiretroviral therapy during primary infection has been associated with dramatic increases and preservation of $\mathrm{CD}^{+} \mathrm{T}$ cell counts while at the same time maintaining HIV-specific immune responses (16). The work of Garber and colleagues suggests that such approaches that seek to limit the deleterious effect of HIV replication on immune activation are worthy of further investigation.

Finally, we believe that the issues raised by the Garber study may explain some puzzling observations pertaining to disease outcomes in patients with multi-drugresistant HIV. Antiretroviral-treated individuals with drug-resistant HIV often experience a durable decrease in viral load below pretreatment levels and a durable increase in $\mathrm{CD}^{+} \mathrm{T}$ cell counts. This sustained immunologic benefit occurs even after controlling for the level of viremia and is associated with increased numbers of HIV-specific CD $4^{+} \mathrm{T}$ cell counts and decreased levels of immune activation (17, 18). Therapeutic manipulation of HIV in which the pathogenic effect of the virus is reduced may be a reasonable approach, particularly in patients for whom fully suppressive combination antiretroviral therapy is not available.

In summary, the work of Garber et al. tells us things we did not know, but also leaves many questions open (9). It shows that immune activation contributes directly to the initial peak viremia, and that the generation of a primary immune response to SIV is a critical determinant of the steady-state level of viremia that occurs during chronic infection. This study also provides preliminary albeit largely indirect evidence that the immunologic response may also be harmful inasmuch as it provides a larger number of target cells to support viral replication. More importantly, this study is one of the first to differentiate the beneficial from the harmful effects of the host response and therefore sets the stage in which strategies might be devised that limit the in vivo pathogenic effect of the virus rather than simply reducing the in vivo replicative fitness of the virus.

Address correspondence to: Bruce D. Walker, Howard Hughes Medical Institute and Partners AIDS Research Center, Massachusetts General Hospital and Division of
AIDS, Harvard Medical School, Boston, Massachusetts 02114, USA. Phone: (617) 724-8332; Fax: (617) 726-4691; E-mail: bwalker@partners.org.

1. Douek, D.C., et al. 2002. HIV preferentially infects HIV-specific CD4 ${ }^{+}$T cells. Nature. 417:95-98.

2. Hellerstein, M.K., et al. 2003. Subpopulations of long-lived and short-lived T cells in advanced HIV-1 infection. J. Clin. Invest. 112:956-966. doi:10.1172/ JCI200317533.

3. Silvestri, G., and Feinberg, M.B. 2003. Turnover of lymphocytes and conceptual paradigms in HIV infection. J. Clin. Invest. 112:821-824. doi:10.1172/ JCI200319799.

4. Grossman, Z., Meier-Schellersheim, M., Sousa, A.E., Victorino, R.M., and Paul, W.E. 2002. CD4 ${ }^{+}$T-cell depletion in HIV infection: are we closer to understanding the cause? Nat. Med. 8:319-323.

5. Liu, Z., et al. 1998. CD8 ${ }^{+}$T-lymphocyte activation in HIV-1 disease reflects an aspect of pathogenesis distinct from viral burden and immunodeficiency. J. Acquir. Immune Defic. Syndr. Hum. Retrovirol. 18:332-340.

6. Hunt, P.W., et al. 2003. T cell activation is associated with lower $\mathrm{CD}^{+} \mathrm{T}$ cell gains in human immunodeficiency virus-infected patients with sustained viral suppression during antiretroviral therapy. J. Infect. Dis. 187:1534-1543.

7. Silvestri, G., et al. 2003. Nonpathogenic SIV infection of sooty mangabeys is characterized by limited bystander immunopathology despite chronic highlevel viremia. Immunity. 18:441-452.

8. Broussard, S.R., et al. 2001. Simian immunodeficiency virus replicates to high levels in naturally infected African green monkeys without inducing immunologic or neurologic disease. J. Virol. 75:2262-2275.

9. Garber, D.A., et al. 2004. Blockade of T cell costimulation reveals interrelated actions of $\mathrm{CD}^{+}$and $\mathrm{CD} 8^{+}$ $\mathrm{T}$ cells in control of SIV replication. J. Clin. Invest. 113:836-845. doi:10.1172/JCI200419442.

10. Rey-Cuille, M.A., et al. 1998. Simian immunodeficiency virus replicates to high levels in sooty mangabeys without inducing disease. J. Virol. 72:3872-3886

11. Chakrabarti, L.A., et al. 2000. Normal T-cell turnover in sooty mangabeys harboring active simian immunodeficiency virus infection. J. Virol. 74:1209-1223.

12. Trachtenberg, E., et al. 2003. Advantage of rare HLA supertype in HIV disease progression. Nat. Med. 9:928-935.

13. Goulder, P.J., et al. 2001. Evolution and transmission of stable CTL escape mutations in HIV infection. Nature. 412:334-338.

14. Moore, C.B., et al. 2002. Evidence of HIV-1 adaptation to HLA-restricted immune responses at a population level. Science. 296:1439-1443.

15. Brander, C., and Walker, B.D. 2003. Gradual adaptation of HIV to human host populations: good or bad news? Nat. Med. 9:1359-1362.

16. Rizzardi, G.P., et al. 2002. Treatment of primary HIV-1 infection with cyclosporin A coupled with highly active antiretroviral therapy. J. Clin. Invest. 109:681-688. doi:10.1172/JCI200214522.

17. Deeks, S.G., et al. 2002. CD4 ${ }^{+} \mathrm{T}$ cell kinetics and activation in human immunodeficiency virus-infected patients who remain viremic despite long-term treatment with protease inhibitor-based therapy. J. Infect. Dis. 185:315-323.

18. Deeks, S.G., et al. 2004. Strong cell-mediated immune responses are associated with the maintenance of low-level viremia in antiretroviral-treated individuals with drug-resistant human immunodeficiency virus type 1. J. Infect. Dis. 189:312-321. 